\title{
Simple and Effective Theory of Movement Steadiness
}

\author{
Smol'yakov Eduard Rimovich \\ Department of Mathematics, Lomonosov Moscow State University, Moscow, Russia
}

Email address:

ser-math@rambler.ru

\section{To cite this article:}

Smol'yakov Eduard Rimovich. Simple and Effective Theory of Movement Steadiness. International Journal of Theoretical and Applied Mathematics. Vol. 5, No. 6, 2019, pp. 113-117. doi: 10.11648/j.ijtam.20190506.15

Received: November 10, 2019; Accepted: December 2, 2019; Published: December 11, 2019

\begin{abstract}
It is proposed the very simple and quick method for estimation of the asymptotic stability of any nonlinear dynamic systems, in particular, of the high-dimensional systems for which Tailor series of the right-hand sides of the differential equations converge very slowly. In such problems, the sum of terms of the order of smallness higher than two can substantially exceed the value of any term of second order. In this case, Lyapunov's methods cannot guarantee correct stability estimate at all. The new method does not use the notion of Liapunov function and, therefore, one has no numerous shortcomings of all Liapunov methods. In this paper, it is proposed to replace the very complex problem of the searching for Liapunov function with a very simple problem of the searching maximum of the function of $\mathrm{n}$ coordinates (that is of the velocity of variation in metrics of the perturbed state space). However, one is not intended for the linear systems.
\end{abstract}

Keywords: Nonlinear Dynamical Systems, Movement Steadiness, New Theory

\section{Introduction}

It is proposed such broadening of the Smol'yakov method $[1,2]$ which permits quickly to solve the question about existence or absence of the asymptotical stability even in very complex cases when the solution could not be found.

The working out of the problem of the movement steadiness began in the end of 19 century in several works of E. Raus, A. E. Jukovskii, A. M. Liapunov and later was continued by N. G. Chetaev, D. R. Merkin, N. N. Krasovskii and others. The most serious results were obtained in the dissertation of A. M. Liapunov in 1892. He proposed the general formulation for the stable motion problem. Unfortunately, this method, based on the sufficient conditions of the stable motion, has many serious shortcomings. In particular, the classical Liapunov's theory often does not permit to find the asymptotical steadiness even if one exists.

The basic Liapunov theorem declares: "If for the differential equation of the perturbed motion it is possible to find a positive-definite function $V(x)>0$, such that the full derivative in time $(\dot{V})$ will be negative-definite $(\dot{V}<0)$ or $\dot{V} \equiv 0$, then non-perturbed motion is stable."

If the last requirement $\dot{V} \equiv 0$ in Liapunov's theorem is excluded, then this theorem defines the asymptotical steadiness. But the last requirement (the pseudo-stability, i.e the stability in means $(V>0, \dot{V} \equiv 0))$ does not define some stability from the practical point of view.

Among shortcomings of the classic theory [3-14], it is necessary to notice, that, firstly, the searching for the desirable function $V(x)$ is very complex, secondly, it is required to expand the perturbed equations in rows, thirdly, method of Liapunov functions is not correct because the different Liapunov functions define absolutely different asymptotical stability sets in any concrete problem (see example 1 in [1]). These shortcomings are the consequence of the fact that Liapunov method is based only on the individual small terms of the first and second order. However, in the nonlinear problems (especially of the great dimension $(n \geq 5)$ ), the particular sums of the higher order terms can considerably exceed any second order term (from which Liapunov function is formed).

Liapunov method is based on the sufficient conditions of the movement steadiness and it is impossible often to find Liapunov function even if the asymptotically stable movement exists in the considered problem. The proposed new method is based on the necessary conditions and one allows always to find the stable motion when it exists.

\section{Statement of the Problem}

Let a process in $n$-space be given by the vector 
differential equation

$$
\frac{d y}{d t}=Y(y(t), t)
$$

where $Y(y, t)$ is the known vector-function satisfying requirements ensuring existence of the solution of the equation (1), $y(t)=\left(y_{1}(t), \ldots, y_{n}(t)\right.$ is the vector-function of the phase-coordinates

$\mathrm{y}_{i}(t), i=1, \ldots n$. And let the partial derivatives $\frac{\partial^{2} Y_{i}}{\partial y_{k}^{2}}, i, k=1, \ldots, n$, exist and ones are continuous.

And let $z(t)$ be some solution of the equation (1). It is required to estimate the steadiness of $z(t)$ with respect to the small perturbations $x(t)$ :

$$
x(t)=y(t)-z(t)
$$

Introduce this equality into equation (1) and rewrite it via coordinates

$$
\frac{d z_{i}}{d t}+\frac{d x_{i}}{d t}=Y_{i}\left(z_{1}+x_{1}, \ldots, z_{n}+x_{n}, t\right), i=1, \ldots, n
$$

The investigation of the steadiness can be performed directly on the basis of the equations (2) or after expansion of these equations in rows on the small parameter $x(t)$ in a neighbourhood of the solution $z(t)$ :

$$
\frac{d z_{i}}{d t}+\frac{d x_{i}}{d t}=Y_{i}(z, t)+\left(\sum_{k-1}^{n} \frac{\partial Y_{i}}{\partial x_{k}}\right)_{z} x_{k}+\Delta Y_{i}, i=1, \ldots, n,
$$

Where $\Delta Y_{i}$ is the sum of the members above the first order. As $z(t)$ is the solution of the equation

$$
\frac{d z}{d t}=Y(z, t)
$$

we receive the following perturbed equations

$$
\frac{d x_{i}}{\mathrm{dt}}=\sum_{k=1}^{n}\left(\frac{\partial Y_{i}}{\partial x_{k}}\right)_{\mathrm{z}} x_{k}+\Delta Y_{i}, i=1, \ldots, n
$$

By means of urge towards $x(t) \rightarrow 0$ in the equations (2) or (4) we can judge about the steadiness of the solution $z(t)$ of the equation (4)

Using only the linear terms of the equations (4) seldom brings success. The analysis of the (4) usually is done by means of the Liapunov functions $V(x)$, [3-14].

\section{The New Method of Stability of the Non-linear Dynamic Systems}

Consider a really different approach to the problem of steadiness based on the variational calculation $[1,2]$ without shortcomings of the classic theory of Liapunov functions.

Let in $n$-space $X$ be defined half-metrics $S=\frac{1}{2} \sum_{k=1}^{n} x_{k}^{2}$ and a small quantity $\varepsilon>0$. Consider the solution of equation (2) (or (4)) in a small $\varepsilon$-environment of zero in $X$ :

$$
S=\frac{1}{2} \sum_{k=1}^{n} x_{k}^{2} \leq \varepsilon .
$$

Definition 1. We say that a solution of the equation (1) is $\mathcal{E}$-stable if there exists the small quantity $\mathcal{E}>0$ and a moment $t_{1}\left(0<t_{1}<\infty\right)$ that for all $t>t_{1}$ the trajectory $x(t)$ remains in the sphere (5). And we say that a movement is asymptotically stable if, for any small quantity $\varepsilon>0$, the trajectory $x(t)$ aspires to zero and reaches value $x(t) \equiv 0$ in (5) for $t \leq \infty$. This means that the movement in the space $X$ satisfies condition $\dot{S}(x)=(\operatorname{grad} \mathrm{S}, \dot{x}) \leq 0$.

Assertion 1. Suppose that the problem of the movement steadiness has a positive solution. As it follows from Definition 1, the object moves inside sphere (5) in the space $X$. In this case $\dot{S}(x)=(\operatorname{grad} \mathrm{S}, \dot{x}) \leq 0$ and function $\dot{S}(x)$ reaches its maximum in some point $x$ in (5) (at the moment $t>t_{1}$ ), and we talk in this case about $\mathcal{E}$-stable. It is obvious that the global maximum in (5) can be reached only in zero of the space $X$. It means that the asymptotical stability has place.

The Assertion 1, in essence, proves the following theorem.

Theorem 1. For a solution of the dynamic system to be $\mathcal{E}$-stable to the small perturbations it is necessary that the full derivative (about time) $\dot{S}(x)$ of the function $S(x)$, calculated with regard to the differential equations (2) or (4), reached maximum in the sphere (4), and in the case of the asymptotical stability, it is necessary that maximum $\dot{S}(x)$ be reached in the point $x=0$.

Consequence 1 . For the asymptotical stability of zerosolution of the equations (2) or (4) relative to the coordinate $x_{i}(t)$, it is necessary that, in any small environment of the point $x=0$ and in this point, the following conditions take place [15]:

$$
\frac{\partial^{2} \dot{\mathrm{S}}}{\partial x_{i}^{2}} \leq 0, \frac{\partial \dot{S}}{\partial x_{i}}=0,(i=1, \ldots, n)
$$

for the asymptotical stability of zero-solution of the equations (2) or (4), it is necessary that, in any small environment of the point $x=0$ and in this point, the conditions (6) be satisfied simultaneously for all $i=1, \ldots, n$.

Proposal 1. We shall speak that, in a small $\mathcal{E}$-environment of zero and in the point $x=0$, the weak asymptotical stability of the zero-solution of equations (2) or (4) relative to the coordinate $x_{k}$ has place if, in the case 


$$
\frac{\partial^{2} \dot{S}}{\partial x_{k}^{2}}=0
$$

the following conditions are satisfied

$$
\frac{\partial}{\partial x_{k}}\left[\frac{d}{d t}\left(\frac{\partial^{2} \dot{S}}{\partial x_{k}^{2}}\right)\right] \geq 0
$$

if the conditions (6) are satisfied for all $i=1, \ldots, n$ and if at least for one coordinate $x_{k}$ are also satisfied the conditions (7) and (8), we shall speak about the week asymptotical stability of the problem (1).

The Theorem 1 and Proposal 1 allow to estimate steadiness of the nonlinear dynamic systems far simpler and quicker than Liapunov's method. The proposed new method does not require the very complex searching for Liapunov's functions and one reduces the stability problem to a very simple problem searching $\max _{x \in R^{n}} \dot{\mathrm{S}}(x)$.

In some cases, theorem 1 and proposal 1 can be also used for investigation of some linear systems as is demonstrated by the example 2 below.

\section{Demonstration of Possibilities of the New Method}

Example 1 [3]. (Satellite on the circular orbit). Estimate the movement steadiness of the satellite on the circular orbit using the following nonlinear differential equations of the perturbation movement:

$$
\begin{gathered}
\frac{d x_{1}}{d t}=x_{2} \\
\frac{d x_{2}}{d t}=\left(r_{o}+x_{1}\right)\left[x_{4}^{2}+\cos ^{2} x_{3}\left(\omega+x_{5}\right)^{2}\right]-\frac{\omega^{2} r_{0}^{3}}{\left(r_{0}+x_{1}\right)^{2}} \\
\frac{d x_{3}}{d t}=x_{4_{4}} \\
\frac{d x_{4}}{d t}=-\frac{2 x_{2} x_{4}}{r_{0}+x_{1}}-\frac{1}{2}\left(\omega+x_{5}\right)^{2} \sin 2 x_{3}
\end{gathered}
$$

$$
\frac{\partial^{2} \dot{S}}{\partial x_{5}^{2}}=2 x_{2}\left(r_{0}+x_{1}\right) \cos ^{2} x_{3}-x_{4} \sin 2 x_{3}-\frac{2 x_{2}}{r_{0}+x_{1}}+2 x_{4} \operatorname{tg} x_{3}-\frac{2 x_{2}}{\left(r_{0}+x_{1}\right)}+2 x_{4} \operatorname{tg} x_{2}
$$

Hence, it is obvious that in any small environment (5) of zero and in the point $x=0$ the second partial derivatives of $\dot{S}$ about coordinates $x_{1}, x_{3}, x_{4}, x_{5}$ can have any sign. It means that the asymptotical stability has no place in this problem. However, for coordinate $x_{2}$ (in point $x=0$ ) we find

$$
\frac{\partial}{\partial x_{2}}\left[\frac{d}{d t}\left(\frac{\partial^{2} \dot{S}}{\partial x_{2}^{2}}\right)\right]>0
$$

$$
\frac{d x_{5}}{d t}=-\frac{2 x_{2}}{\left(r_{0}+x_{1}\right)}\left(\omega+x_{5}\right)+2 x_{4}\left(\omega+x_{5}\right) \operatorname{tg} x_{3}
$$

The order of this system is $n=5$. Therefore, the sum of the third order terms can be higher than any term of the second order in Liapunov function. In this case, there are doubts about Liapunov method truthfulness. In [3], by Liapunov methods, it was received only pseudo-stability of the satellite (i.e the stability on the pair $(V>0, \dot{V} \equiv 0)$ ), but the asymptotical stability or its absence was not proved. The proposed method proves the absence of the asymptotical stability.

Introduce the function

$$
\begin{gathered}
\dot{S}=\sum_{i=1}^{5} x_{i} \dot{x}_{i}=x_{1} x_{2}+x_{2} \\
\left\{\left(r_{o}+x_{1}\right)\left[x_{4}^{2}+\cos ^{2} x_{3}\left(\omega+x_{5}\right)^{2}\right]-\frac{\omega^{2} r_{0}^{3}}{\left(r_{0}+x_{1}\right)^{2}}\right\}+ \\
x_{3} x_{4}-x_{4}\left\{\frac{2 x_{2} x_{4}}{r_{0}+x_{1}}+\frac{1}{2}\left(\omega+x_{5}\right)^{2} \sin 2 x_{3}\right\}+ \\
x_{5}\left\{-\frac{2 x_{2}}{\left(r_{0}+x_{1}\right)}\left(\omega+x_{5}\right)+2 x_{4}\left(\omega+x_{5}\right) \operatorname{tg} x_{3}\right\} .
\end{gathered}
$$

Calculating the second partial derivatives of $\dot{S}$, we receive

$$
\begin{gathered}
\frac{\partial^{2} \dot{S}}{\partial x_{1}^{2}}=-\frac{x_{2}}{\left(r_{0}+x_{1}\right)^{3}}\left\{\frac{6 \omega^{2} r_{0}^{3}}{\left(r_{0}+x_{1}\right)}+4 x_{4}^{2}+4 x_{5}\left(\omega+x_{5}\right)\right\}, \\
\frac{\partial^{2} \dot{S}}{\partial x_{2}^{2}}=0, \\
\frac{\partial^{2} \dot{S}}{\partial x_{3}^{2}}=-2 x_{2}\left(r_{0}+x_{1}\right)\left(\omega+x_{5}\right)^{2} \cos 2 x_{3}+4 x_{4} x_{5}\left(\omega+x_{5}\right) \frac{\sin x_{3}}{\cos ^{3} x_{3}},(10) \\
\frac{\partial^{2} \dot{S}}{\partial x_{4}^{2}}=2 x_{2}\left(r_{0}+x_{1}\right)-4 \frac{x_{2}}{\left(r_{0}+x_{1}\right)},
\end{gathered}
$$

that is, only for the coordinate $x_{2}$ we receive the weak asymptotical stability.

Example 2 [3] (Gyrocompass). Let the gyroscope be set in the horizontal plane and angles $\alpha$ and $\beta$ define the deviations of the gyroscope axes from the vertical, and let $k \alpha$ and $k \beta$ be some renovation momentums, where $k>0$. Estimate the steadiness of gyrocompass using the following linear model of the movement: 


$$
\begin{aligned}
& J \ddot{\alpha}+b \dot{\alpha}-H \dot{\beta}-k \beta=0, \\
& J \ddot{\beta}+b \dot{\beta}+H \dot{\alpha}+k \alpha=0,
\end{aligned}
$$

where $J$ is the equatorial moment of inertia of the gyroscope, $H$ is the kinetic moment and $b$ is the coefficient of the resistance forces.

Assuming $\quad \alpha=x_{1}, \dot{\alpha}=\dot{x}_{1}=x_{2}, \beta=x_{3}, \dot{\beta}=\dot{x}_{3}=x_{4}, \quad$ we receive the following equations

$$
\begin{aligned}
& \dot{x}_{1}=x_{2}, \dot{x}_{2}=-\bar{b} x_{2}+\bar{H} x_{4}+\bar{k} x_{3}, \\
& \dot{x}_{3}=x_{4}, 2 \dot{x}_{4}=-\bar{b} x_{4}-\bar{H} x_{2}-\bar{k} x_{1} .
\end{aligned}
$$

Introduce function $S=(\operatorname{grad} S, \dot{x})$ :

$$
\dot{S}=x_{1} x_{2}-\bar{b} x_{2}^{2}+\bar{H} x_{2} x_{4}+\bar{k} x_{2} x_{3}+x_{3} x_{4}-\bar{b} x_{4}^{2}-\bar{H} x_{2} x_{4}-\bar{k} x_{1} x_{4}
$$

and calculate the second particular derivatives

$$
\frac{\partial^{2} \dot{S}}{\partial x_{1}^{2}}=\frac{\partial^{2} \dot{S}}{\partial x_{3}^{2}}=0, \frac{\partial^{2} \dot{S}}{\partial x_{2}^{2}}=\frac{\partial^{2} \dot{S}}{\partial x_{4}^{2}}=-2 \bar{b}<0 .
$$

From (13), it follows that the system (12) is asymptotically stable about only the angular speeds $x_{2}$ and $x_{4}$. For estimation of stability about angles $\alpha, \beta$, it is necessary to use Proposal 1:

$$
\frac{\partial}{\partial x_{1}}\left(\frac{d}{d t}\left(\frac{\partial \dot{\mathrm{S}}}{\partial x_{1}}\right)\right)=\frac{\partial}{\partial x_{3}}\left(\frac{d}{d t}\left(\frac{\partial \dot{\mathrm{S}}}{\partial x_{3}}\right)\right)=\bar{k}^{2}>0,
$$

hence it follows only the weak asymptotical stability about these angles.

In [2, c. 211-213], it was found the asymptotical stability of the system (12) for all coordinates, whereas we receive the weaker asymptotical stability for angles than for the angular speeds. Perhaps, our assertion is more exact.

Example 3 [3] (Cone pendulum). Consider the idealized model of a pendulum spinning about the vertical axis. Le $l$ be length of the pendulum thread, let $g$ denote the gravitational constant, let $\theta$ and $\psi$ denote, respectively, an angle of decline from the vertical axis and angle of rotation in the horizontal plane. The pendulum movement is described by the following non-linear differential equations, [3]:

$$
\ddot{\theta}=-\frac{\mathrm{g}}{1} \sin \theta+\psi^{2} \sin \theta \cos \theta, \ddot{\psi}=-2 \dot{\theta} \dot{\psi} \operatorname{ctg} \theta .
$$

Introducing the new variables $\theta=y_{1}, \dot{\theta}=y_{2}, \dot{\psi}=y_{3}$, we receive the following system of the third non-linear differential equations

$$
\begin{aligned}
& \dot{y}_{1}=y_{2}, \\
& y_{2}=-\frac{g}{l} \sin y_{1}+y_{3}^{2} \sin y_{1} \cos y_{1}, \\
& \dot{y}_{3}=-2 y_{2} y_{3} \operatorname{ctg} y_{1} .
\end{aligned}
$$

Investigate the stability of the following movement

$$
\theta=y_{1}=\alpha=\text { const }, \dot{\theta}=y_{2}=0, \dot{\psi}=y_{3}=\omega=\text { const } \text {. }
$$

Placing these values in (14), we find

$$
\omega^{2} \cos \alpha=\frac{\mathrm{g}}{1} .
$$

Introducing the new coordinates $y_{1}=\alpha+x_{1}, y_{2}=x_{2}, y_{3}=\omega+x_{3}$, and taking into account (15), we receive the following perturbed motion equations

$$
\begin{aligned}
& \dot{x}_{1}=x_{2}, \\
& \dot{x}_{2}=-\omega^{2} \cos \alpha \sin \left(\alpha+x_{1}\right)+\left(\omega+x_{3}\right)^{2} \sin \left(\alpha+x_{1}\right) \cos \left(\alpha+x_{1}\right), \\
& \dot{x}_{3}=-2 x_{2}\left(\omega+x_{3}\right) \operatorname{ctg}\left(\alpha+x_{1}\right)
\end{aligned}
$$

The asymptotical stability or its absence were not established for the system (16) in [3].

Prove that the asymptotical stability has no place in the problem (16). Consider function

$$
\begin{aligned}
& \dot{S}=x_{1} x_{2}+x_{2}\left[-\omega^{2} \cos \alpha \sin \left(\alpha+x_{1}\right)+\left(\omega+x_{3}\right)^{2} \sin \left(\alpha+x_{1}\right) \cos \left(\alpha+x_{1}\right)\right]+ \\
& x_{3}\left[-2 x_{2}\left(\omega+x_{3}\right) \operatorname{ctg}\left(\alpha+x_{1}\right)\right]
\end{aligned}
$$

and calculate its the second particular derivatives.

We receive $\frac{\partial^{2} \dot{S}}{\partial x_{2}^{2}}=0$ and the inequality (11) is not satisfied for the coordinate $x_{2}$. This means that the variable $x_{2}$ is not even weak asymptotical stable. The second particular derivatives about $x_{1}$ and $x_{3}$ (which are not given here in consequence of their bigness) are the homogeneous linear functions about the co-ordinate $x_{2}$ which can be of any sign in any environment of zero. Hence, it follows absence of the asymptotical stability about values $x_{1}$ and $x_{3}$.

Notice. The proposed new method does not require expanding of the perturbed differential equations in rows and permits to receive the function $\dot{S}$ directly on the basis of the equations

$$
\frac{d x_{i}}{d t}=Y_{i}\left(z_{1}+x_{1}, \ldots, z_{n}+x_{n}, t\right)-\frac{d z_{i}}{d t}, i=1, \ldots, n .
$$




\section{Conclusion}

The new method does not use Liapunov functions. As it was demonstrated by the examples, this new method is far simpler and more effective than the classic Liapunov theory and all its known modifications and improvements [3-14]. Engineers now can in some minutes or hours define the asymptotical stability or absence of the stability in any nonlinear dynamical systems, while up to now Liapunov methods required often many hours, months or did not permit to estimate the stability at all.

\section{Acknowledgements}

The work was supported by the Russian Foundation for Basic Research (grant no. 18-01-00842-a).

\section{References}

[1] Smol' yakov E. R. Absolutely New, Simple and Effective Theory of Movement Steadiness//International Journal of Theoretical and Applied Mathematics, 2018, Vo. 4, no. 4, pp. 35-39.

[2] Smol'yakov E. R. An Effective Method of Stability Analysis for Highly Nonlinear Dynamic Systems// Cybernetics and Systems Analysis, 2019, Vol. 55, no. 4, pp. 15-23.

[3] Merkin D. A. Introduction into theory of movement stability. Moscow: Nauka, 1987.
[4] Liapunov A. M. "Collected works", Vol. 1-5, MoscowLeningrad (1954-1965).

[5] Routh E. J. The Advanced Part of a treatise on the Dynamics of a System of rigid bodies. London, 1884.

[6] Ziegler H. Linear Elastic Stability. Critical Analysis of Methods// ZAMP. Basel - Zurich, IV, F-2. 1953.

[7] Hagedorn P. Uber die instabilitat konservativer systeme mit girosropischen Kraften//RationalMech and Anal. 1975, Bd. 58, № 1 .

[8] Herrman G. Stability of Equilibrium of Elastic Systems Subjested to Nonconservative Fordes//Applied Mechanics Reviews. 1967. V.20. № 2.

[9] Karapetyan A. V. Stability of Stationary movements. Moscow: URSS, 1998.

[10] Chetaev N. G. Stability of movement. The works on the analytical mechanics. Moscow: RAN SSSR, 1962.

[11] Krasovskii N. N. Some problems of the movement stability theory. Moscow: Fizmatgis, 1959.

[12] Demidovich B. P. Lecture on mathematical theory of stability. Moscow: MGU, 1998.

[13] La Salle J., Lefschetz S., Stability by Lyapunov's direct method with applications, Acad. Press (1961).

[14] Lin C. C., The theory of hydrodynamic stability, Cambridge Univ. Press (1955).

[15] Bliss G. A. Lecture on the variational calculation.. Moscow.: IL, 1950. 